\title{
A method for fault diagnosability evaluation of spacecraft control system
}

\author{
Chen Yin, Zhangming He, Jiongqi Wang and Haiyin Zhou \\ Science College National University of Defense Technology, China \\ *yinchen_register@hotmail.com
}

Keywords: Fault Diagnosability Evaluation, Spacecraft Control Systems, Energy-statistics

\begin{abstract}
The diagnosability is the inherent property of spacecraft control system, mainly including the fault detectability and isolability. Analysis of diagnosability in the system design stage contributes to reduce the failure risks for it reveal the relation between system structure and faults, which suggests a easier way to design a fault diagnosis algorithm and devoting to a necessary redundancy and safety. The model uncertainties and process noise affect the diagnosability evaluation but still gain deficient attention in current researches, in this work we consider a method using Energy-statistics to evaluate the system diagnosability with model uncertainties as well as process noise, propose the definition and the calculation of fault detectability and isolability.
\end{abstract}

\section{Introduction}

Modern spacecraft is required to be multi-mission available despite high risks, high cost and long cycles, which suggests that the spacecraft control system is critical to keep spacecraft working in staple state and coping with complex natural conditions in outer space. Moreover, once launched the flexibility remains limited. Coping with faults of spacecraft control system effectively can ensure the success of mission and lower the total cost. Currently the researches mainly focus on the diagnosis methods for spacecraft control system faults [1], while fault diagnosis method require the system to be diagnosable.

Spacecraft control system faults can only be detected via diagnosis methods when the system diagnosability can be met [2], which proves the significance of diagnosability analysis in the system design stage. Better diagnosability of a system contributes to easier fault diagnosis algorithm and higher efficiency, which plays a constructive in dealing with on-orbit faults. The definition of diagnosability is: when single or more faults occur, the ability to diagnosis the fault, including detectability and isolability [3].

\section{System Formulation and Problem Statement}

The Concept of Diagnosability A fault on a component of the system is said to be detectable if knowledge of system inputs and outputs over a finite time interval following the occurrence of the fault allows the detection in spite of disturbances [3].

System Formulation. The spacecraft control system can be treat as a discrete-time descriptor model in the form

$$
\left\{\begin{array}{r}
\mathbf{x}(k+1)=\mathbf{A x}(k)+\mathbf{B}_{u} \mathbf{u}(k)+\mathbf{B}_{f} \mathbf{f}(k)+\mathbf{B}_{w} \mathbf{w}(k) \\
\mathbf{y}(k)=\mathbf{C x}(k)+\mathbf{D}_{u} \mathbf{u}(k)+\mathbf{D}_{f} \mathbf{f}(k)+\mathbf{D}_{v} \mathbf{v}(k)
\end{array}\right.
$$

In formula (1), $\mathbf{x} \in \mathbb{R}^{s}$ denotes the vector that contains state variables, $\mathbf{y} \in \mathbb{R}^{m}$ is the system output, $\mathbf{u} \in \mathbb{R}^{q}$ is the system input, $\mathbf{f} \in \mathbb{R}^{p}$ is the vector that contains fault variables. $\mathbf{w} \in \mathbb{R}^{l}$ represents the process noise, $\mathbf{v} \in \mathbb{R}^{t}$ is the observation noise. $\mathbf{w}$ and $\mathbf{v}$ are i.i.d. Gaussian random vectors with zero mean and symmetric positive definite covariance matrices $\boldsymbol{\Sigma}_{w}{ }^{l \times l}$ and $\boldsymbol{\Sigma}_{v}{ }^{1 \times t}$, which demonstrate the model uncertainties and process noise.

With our purpose to conduct the analysis of diagnosability more directly and efficiently, we rewrite the system formulation (1) using a sliding window model [4] of length $n$ : 


$$
\begin{aligned}
& \mathbf{z}=\left(\mathbf{y}[t-n+1]^{T}, \ldots, \mathbf{y}[t]^{T}, \mathbf{u}[t-n+1]^{T}, \ldots, \mathbf{u}[t]^{T}\right)^{T}, \quad \mathbf{x}=\left(\mathbf{x}[t-n+1]^{T}, \ldots, \mathbf{x}[t], \mathbf{x}[t+1]^{T}\right)^{T} \\
& \mathbf{f}=\left(\mathbf{f}[t-n+1]^{T}, \ldots, \mathbf{f}[t]\right)^{T}, \quad \mathbf{e}=\left(\mathbf{w}[t-n+1]^{T}, \ldots, \mathbf{w}[t]^{T}, \mathbf{v}[t-n+1]^{T}, \ldots, \mathbf{v}[t]^{T}\right)^{T}
\end{aligned}
$$

Where $\mathbf{z} \in \mathbb{R}^{n(m+q)}, \mathbf{x} \in \mathbb{R}^{(n+1) s}, \mathbf{f} \in \mathbb{R}^{n p}$, e is a stochastic vector with zero mean, $\mathbf{e} \in \mathbb{R}^{n(l+t)}$.

Then we get the rewritten form of (1):

$$
\mathbf{L z}=\mathbf{H x}+\mathbf{F f}+\mathbf{E e}
$$

In (3) there exist

$$
\begin{aligned}
& \mathbf{L}=\left[\begin{array}{cccccccc}
\mathbf{0} & \mathbf{0} & \cdots & \mathbf{0} & -\mathbf{B}_{u} & \mathbf{0} & \ldots & \mathbf{0} \\
\mathbf{0} & \mathbf{0} & & \mathbf{0} & \mathbf{0} & -\mathbf{B}_{u} & & \mathbf{0} \\
\vdots & & \ddots & \vdots & \vdots & & \ddots & \vdots \\
\mathbf{0} & \mathbf{0} & \cdots & \mathbf{0} & \mathbf{0} & \cdots & \mathbf{0} & -\mathbf{B}_{u} \\
\mathbf{I} & \mathbf{0} & \cdots & \mathbf{0} & \mathbf{D}_{u} & \mathbf{0} & \ldots & \mathbf{0} \\
\mathbf{0} & \mathbf{I} & & \mathbf{0} & \mathbf{0} & \mathbf{D}_{u} & & \mathbf{0} \\
\vdots & & \ddots & \vdots & \vdots & & \ddots & \vdots \\
\mathbf{0} & \mathbf{0} & \cdots & \mathbf{I} & \mathbf{0} & \cdots & \mathbf{0} & \mathbf{D}_{u}
\end{array}\right], \quad \mathbf{H}=\left[\begin{array}{ccccc}
\mathbf{A} & -\mathbf{I} & \mathbf{0} & \cdots & \mathbf{0} \\
\mathbf{0} & \mathbf{A} & -\mathbf{I} & \cdots & \mathbf{0} \\
\vdots & \ddots & \ddots & \ddots & \vdots \\
\mathbf{0} & \cdots & \mathbf{0} & \mathbf{A} & -\mathbf{I} \\
\mathbf{C} & \mathbf{0} & \mathbf{0} & \ldots & \mathbf{0} \\
\mathbf{0} & \mathbf{C} & \mathbf{0} & \ldots & \mathbf{0} \\
\vdots & \ddots & \ddots & \ddots & \vdots \\
\mathbf{0} & \cdots & \mathbf{0} & \mathbf{C} & \mathbf{0}
\end{array}\right], \\
& \mathbf{F}=\left[\begin{array}{ccccc}
\mathbf{B}_{f} & \mathbf{0} & \mathbf{0} & \cdots & \mathbf{0} \\
\mathbf{0} & \mathbf{B}_{f} & \mathbf{0} & \cdots & \mathbf{0} \\
\vdots & \ddots & \ddots & \vdots \\
\mathbf{0} & \cdots & \mathbf{0} & \mathbf{B}_{f} \\
\mathbf{D}_{f} & \mathbf{0} & \mathbf{0} & \cdots & \mathbf{0} \\
\mathbf{0} & \mathbf{D}_{f} & \mathbf{0} & \cdots & \mathbf{0} \\
\vdots & \ddots & \ddots & \vdots \\
\mathbf{0} & \cdots & \mathbf{0} & \mathbf{D}_{f}
\end{array}\right] \quad \mathbf{E}=\left[\begin{array}{ccccccccc}
\mathbf{B}_{w} & \mathbf{0} & \cdots & \mathbf{0} & \mathbf{0} & \mathbf{0} & \cdots & \mathbf{0} \\
\mathbf{0} & \mathbf{B}_{w} & & \mathbf{0} & \mathbf{0} & \mathbf{0} & & \mathbf{0} \\
\vdots & & \ddots & \vdots & \vdots & & \ddots & \vdots \\
\mathbf{0} & \mathbf{0} & \cdots & \mathbf{B}_{w} & \mathbf{0} & \cdots & \mathbf{0} & \mathbf{0} \\
\mathbf{0} & \mathbf{0} & \cdots & \mathbf{0} & \mathbf{D}_{v} & \mathbf{0} & \cdots & \mathbf{0} \\
\mathbf{0} & \mathbf{0} & & \mathbf{0} & \mathbf{0} & \mathbf{D}_{v} & & \mathbf{0} \\
\vdots & & \ddots & \vdots & \vdots & & \ddots & \vdots \\
\mathbf{0} & \mathbf{0} & \cdots & \mathbf{0} & \mathbf{0} & \cdots & \mathbf{0} & \mathbf{D}_{v}
\end{array}\right] .
\end{aligned}
$$

The we multiply (3) on the left with the orthonormal basis $\mathbf{N}_{H}$ for the left-null space of $\mathbf{H}$, this formed

$$
\mathbf{N}_{H} \mathbf{L z}=\mathbf{N}_{H} \mathbf{H x}+\mathbf{N}_{H} \mathbf{F f}+\mathbf{N}_{H} \mathbf{E e}
$$

Since $\mathbf{N}_{H} \mathbf{H x}$ is equal to null matrix, we have $\mathbf{N}_{H} \mathbf{L z}=\mathbf{N}_{H} \mathbf{F f}+\mathbf{N}_{H} \mathbf{E e}$

Form (5) shows that the system performance is influenced by fault vector $\mathbf{f}$ and noise $\mathbf{e}$.

\section{An Application of Energy-statistics to system diagnosability Analysis}

Overview of Energy Distance. Energy distance is a statistical distance between the distributions of random vectors, which characterizes equality of distributions [6].

Definition 1 (Energy distance). The energy distance between the d-dimensional independent random variables $X$ and $Y$ is defined as

$$
\varepsilon(X, Y)=2 E|X-Y|_{d}-E\left|X-X^{\prime}\right|_{d}-E\left|Y-Y^{\prime}\right|_{d}
$$

Where $E|X|_{d}<\infty, E|Y|_{d}<\infty, X^{\prime}$ is an iid copy of $X$, and $Y^{\prime}$ is an iid copy of $Y$. If not specially mentioned, we omit the subscript $d$ for its meaning is very clear.

Definition 2. The two sample energy statistic corresponding to the energy distance $\varepsilon(X, Y)$, for independent random samples $X=X_{1}, \ldots, X_{n_{1}}$ and $Y=Y_{1}, \ldots, Y_{n_{1}}$, there is

$$
\begin{aligned}
\varepsilon_{n_{1}, n_{2}}(X, Y)= & \frac{2}{n_{1} n_{2}} \sum_{i=1}^{n_{1}} \sum_{m=1}^{n_{2}}\left|X_{i}-Y_{m}\right| \\
& -\frac{1}{n_{1}{ }^{2}} \sum_{i=1}^{n_{1}} \sum_{j=1}^{n_{1}}\left|X_{i}-X_{j}\right|-\frac{1}{n_{2}{ }^{2}} \sum_{l=1}^{n_{2}} \sum_{m=1}^{n_{2}}\left|Y_{l}-Y_{m}\right|
\end{aligned}
$$


The statistic $T_{n_{1}, n_{2}}=\frac{n_{1} n_{2}}{n_{1}+n_{2}} \varepsilon_{n_{1}, n_{2}}$ can be applied for testing homogeneneity (equality for distributions of $X$ and $Y$ ). The hypothesis of equality is rejected for large $T_{n_{1}, n_{2}}$.

Proposition 1. The energy distance between $\mathbf{N}_{H} \mathbf{F f}$ and $\mathbf{N}_{H} \mathbf{F f}$ equals to the energy distance between $\mathbf{N}_{H} \mathbf{L} \mathbf{z}_{i}$ and $\mathbf{N}_{H} \mathbf{L z} z_{j}$, that is

$$
\varepsilon\left(\mathbf{N}_{H} \mathbf{L z}, \mathbf{N}_{H} \mathbf{L z} \mathbf{z}_{j}\right)=\varepsilon\left(\mathbf{N}_{H} \mathbf{F f _ { i }}, \mathbf{N}_{H} \mathbf{F f} \mathbf{f}_{j}\right)
$$

Proof: In Definition 1, we have shown the definition of energy distance between two d-dimensional independent random variables $X$ and $Y$ as the form (6):

Then we can derive the conclusion:

$$
\begin{aligned}
& \varepsilon\left(\mathbf{N}_{H} \mathbf{L} \mathbf{z}_{i}, \mathbf{N}_{H} \mathbf{L} \mathbf{z}_{j}\right) \\
& =\varepsilon\left(\mathbf{N}_{H} \mathbf{F f} \mathbf{f}_{i}+\mathbf{N}_{H} \mathbf{E e}, \mathbf{N}_{H} \mathbf{F f} \mathbf{f}_{j}+\mathbf{N}_{H} \mathbf{E e}\right) \\
& =2 E\left|\mathbf{N}_{H} \mathbf{F} \mathbf{f}_{i}+\mathbf{N}_{H} \mathbf{E e}-\mathbf{N}_{H} \mathbf{F f} \mathbf{f}_{j}-\mathbf{N}_{H} \mathbf{E e}\right|-E\left|\left(\mathbf{N}_{H} \mathbf{F} \mathbf{f}_{i}+\mathbf{N}_{H} \mathbf{E e}\right)-\left(\mathbf{N}_{H} \mathbf{F} \mathbf{f}_{i}+\mathbf{N}_{H} \mathbf{E e}\right)^{\prime}\right|-E\left|\left(\mathbf{N}_{H} \mathbf{F f} \mathbf{f}_{j}+\mathbf{N}_{H} \mathbf{E e}\right)-\left(\mathbf{N}_{H} \mathbf{F f} \mathbf{f}_{j}+\mathbf{N}_{H} \mathbf{E e}\right)^{\prime}\right| \\
& =2 E\left|\mathbf{N}_{H} \mathbf{F f} \mathbf{f}_{i} \mathbf{N}_{H} \mathbf{F f}{ }_{j}\right|-E\left|\mathbf{N}_{H} \mathbf{L z} \mathbf{z}_{i}-\left(\mathbf{N}_{H} \mathbf{L z}_{i}\right)^{\prime}\right|-E\left|\mathbf{N}_{H} \mathbf{F f}{ }_{j}-\left(\mathbf{N}_{H} \mathbf{F f}\right)^{\prime}\right| \\
& =\varepsilon\left(\mathbf{N}_{H} \mathbf{F f} \mathbf{N}_{H} \mathbf{F} \mathbf{f}_{j}\right)
\end{aligned}
$$

Definition 3 (Isolability). Let $\mathbf{f}_{i, t}$ corresponds to the value of $\mathbf{f}_{i}$ at the time $t$. Then we select $\mathbf{F}_{i}=\mathbf{f}_{i, t_{1}}, \ldots, \mathbf{f}_{i, t_{m}}, \mathbf{F}_{j}=\mathbf{f}_{j, t_{1}}, \ldots, \mathbf{f}_{j, t_{n}}$, refer to the Definition 3 and proposition 1, we can derive the form of Diagnosability between $\mathbf{F}_{i}$ and $\mathbf{F}_{j}$ as the statistic $T_{m, n}$ :

$$
\begin{aligned}
T_{m, n}= & \frac{m n}{m+n}\left(\frac{2}{m n} \sum_{a=1}^{m} \sum_{b=1}^{n}\left|\mathbf{N}_{H} \mathbf{L} \mathbf{z}_{i, t_{a}}-\mathbf{N}_{H} \mathbf{L} \mathbf{z}_{j, t_{b}}\right|\right. \\
& \left.-\frac{1}{m^{2}} \sum_{c=1}^{m} \sum_{d=1}^{m}\left|\mathbf{N}_{H} \mathbf{L} \mathbf{z}_{i, t_{c}}-\mathbf{N}_{H} \mathbf{L} \mathbf{z}_{i, t_{d}}\right|-\frac{1}{n^{2}} \sum_{e=1}^{n} \sum_{f=1}^{n}\left|\mathbf{N}_{H} \mathbf{L} \mathbf{z}_{j, t_{e}}-\mathbf{N}_{H} \mathbf{L} \mathbf{z}_{j, t_{f}}\right|\right)
\end{aligned}
$$

Definition 4 (detectability). The detectability of fault $\mathbf{F}_{i}$ can be described as the form:

$$
\begin{aligned}
T_{m}= & \frac{m^{2}}{2 m}\left(\frac{2}{m^{2}} m \sum_{a=1}^{m}\left|\mathbf{N}_{H} \mathbf{L} \mathbf{z}_{i, t_{a}}\right|\right. \\
& \left.-\frac{1}{m^{2}} \sum_{c=1}^{m} \sum_{d=1}^{m}\left|\mathbf{N}_{H} \mathbf{L} \mathbf{z}_{i, t_{c}}-\mathbf{N}_{H} \mathbf{L} \mathbf{z}_{i, t_{d}}\right|\right) \\
= & \sum_{a=1}^{m}\left|\mathbf{N}_{H} \mathbf{L} \mathbf{z}_{i, t_{a}}\right|-\frac{1}{2 m} \sum_{c=1}^{m} \sum_{d=1}^{m}\left|\mathbf{N}_{H} \mathbf{L} \mathbf{z}_{i, t_{c}}-\mathbf{N}_{H} \mathbf{L} \mathbf{z}_{i, t_{d}}\right|
\end{aligned}
$$

\section{Case Studies}

In order to state the effectiveness of the proposed method of system diagnosability evaluation, we make a simulation of "infrared earth sensor(IRES) \& gyroscope " [5].

IERS and gyroscope are vital to spacecraft attitude control system. When the spacecraft is at the three-axis stabilized state, let $\varphi$ be the roll angle corresponding to orbit coordinate system, $\theta$ be the elevation angle, $\psi$ be the azimuth angle. $\omega_{0}$ is the angular velocity and $\mathbf{g}(t)$ means the output of the gyroscope, $\mathbf{b}(t)$ is the constant drift, $\mathbf{d}(t)$ is the exponential drift. $\mathbf{n}(t)$ is white noise with Gaussian distribution, $\mathbf{f}_{g}(t)$ is the fault vector of the gyroscope. $\tau_{i}$ is the time constant, $n_{d i}(t)$ and $n_{b i}(t)$ are virtual white noise.Similarly $n_{h \theta}(t)$ and $n_{h \varphi}(t)$ are Gaussian white noises, $f_{h \varphi}(t)$ and $f_{h \theta}(t)$ represent the fault vector of IERS.

Since the attitude angle $\&$ angular velocity is decoupling with the roll/yaw axes, the system can be combined and rewritten as the form 


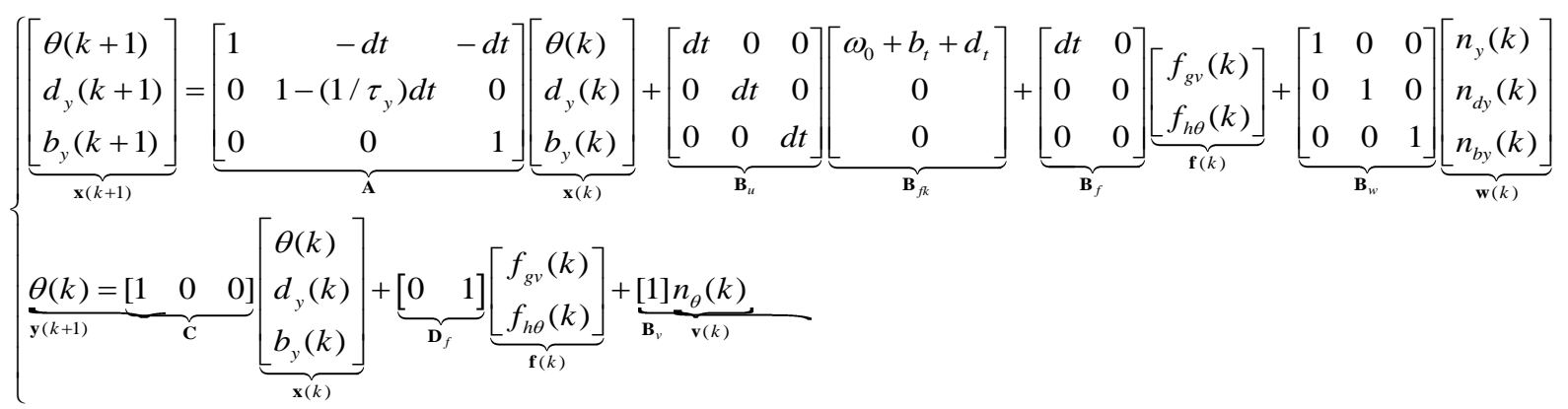

In (12) we assumed the values $d t=0.1 s, \tau_{y}=1, \omega_{0}=0.06 \mathrm{rad} / \mathrm{s}, n_{y} \sim N\left(0,10^{-6}\right)$,

$$
n_{b y} \sim N\left(0,10^{-4}\right), n_{d y} \sim N\left(0,10^{-5}\right), n_{\theta} \sim N\left(0,10^{-4}\right), f_{i} \in\{0,1\} \text {. }
$$

TABLE I THE ISOLABILITY AND DIAGNOSABILITY OF FAULTS

\begin{tabular}{|c|c|c|c|}
\hline \multirow{2}{*}{} & \multicolumn{2}{|c|}{ Isolability } & \multirow{2}{*}{ Diagnosability } \\
\cline { 2 - 3 } & $f_{g v}$ & $f_{h \theta}$ & \\
\hline$f_{g v}$ & 0 & 7.943677 & 6.336015 \\
\hline$f_{h \theta}$ & 7.943677 & 0 & 0.3482534 \\
\hline
\end{tabular}

\section{Conclusions}

In this paper, we clarify the concept of diagnosability and isolability of spacecraft attitude control system and rewrite the system formulation which combines the output values with input values. On the basis of the rewritten model, we propose a new way using the energy statistics to deal with the quantitative analysis of diagnosability and isolability.

\section{References}

[1]Patton, R. J., et al. "Reliable fault diagnosis scheme for a spacecraft attitude control system." Proceedings of the Institution of Mechanical Engineers, Part O: Journal of Risk and Reliability 222.2 (2008): 139-152.

[2]Jan M. Kościelny, et al. "Actuator fault distinguishability study for the DAMADICS benchmark problem." Control Engineering Practice 14.6(2006):645-652.

[3]Liu, Wenjing, C. Liu, and N. Wang. "Overview of Fault Diagnosability Evaluation and Design." Aerospace Control (2011).

[4]Eriksson, Daniel, E. Frisk, and M. Krysander. "A method for quantitative fault diagnosability analysis of stochastic linear descriptor models $\hat{z}$." Automatica 49.6(2013):1591-1600.

[5]Li, Wenbo, D. Wang, and C. Liu. "Fault diagnosability evaluation for spacecraft control systems via direction similarity." Control Conference IEEE, 2014.

[6]Székely, Gábor J., and M. L. Rizzo. "Energy statistics: A class of statistics based on distances." Journal of Statistical Planning \& Inference 143.8(2013):1249-1272. 